\title{
ASYMPTOTIC BEHAVIOR OF A NONLOCAL DIFFUSIVE LOGISTIC EQUATION*
}

\author{
ARNAUD DUCROT ${ }^{\dagger}$ AND PIERRE MAGAL $^{\dagger}$
}

\begin{abstract}
The long time behavior of a logistic-type equation modeling the motion of cells is investigated. The equation we consider takes into account birth and death processes using a simple logistic effect as well as a nonlocal motion of cells using a nonlocal Darcy's law with regular kernel. Using the periodic framework we first investigate the well-posedness of the problem before deriving some information about its long time behavior. The lack of asymptotic compactness of the system is overcome by making use of the Young measure theory. This allows us to conclude that the semiflow converges for the Young measure topology.
\end{abstract}

Key words. nonlocal interactions, asymptotic behavior, Young measures

AMS subject classifications. 37N25, 35L60, 34D05, 34G20

DOI. $10.1137 / 130922100$

1. Introduction. This work is concerned with the study of the integro-differential equation

$$
\left\{\begin{array}{l}
\partial_{t} u(t, x)+\operatorname{div}(u(t, x) \mathbf{J}[u(t, .)](x))=f(u(t, x)), t>0, x \in \mathbb{R}^{N} \\
\mathbf{J}[u(t, .)](x)=-\nabla(K \circ u(t, .))(x), \\
u(t, x+2 \pi k) \equiv u(t, x) \forall t>0, x \in \mathbb{R}^{N}, k \in \mathbb{Z}^{N}
\end{array}\right.
$$

Here $\circ$ denotes the convolution operator on the $N$-dimensional torus $\mathbb{T}^{N}=\mathbb{R}^{N} / 2 \pi \mathbb{Z}^{N}$ defined for each $\mathbb{T}^{N}$-periodic and measurable functions $f$ and $g$ by

$$
(f \circ g)(x)=\left|\mathbb{T}^{N}\right|^{-1} \int_{\mathbb{T}^{N}} f(x-y) g(y) d y,
$$

whenever the above expression makes sense for a.e. $x \in \mathbb{T}^{N}$. Equation (1.1) is supplemented together with some initial datum

$$
u(0, x)=u_{0}(x) \text { with } u_{0} \text { nonnegative and } \mathbb{T}^{N} \text {-periodic function. }
$$

Problem (1.1) arises when looking at spatially periodic solutions of the integrodifferential equation

$$
\left\{\begin{array}{l}
\partial_{t} u(t, x)+\nabla \cdot(u(t, x) \mathbf{v}(t, x))=f(u(t, x)), t>0, x \in \mathbb{R}^{N}, \\
\mathbf{v}(t, x)=-\nabla\left(\int_{\mathbb{R}^{N}} \rho(x-y) u(t, y) d y\right),
\end{array}\right.
$$

associated to a $\mathbb{T}^{N}$-periodic initial datum and fast decaying kernel function $\rho$. Indeed, in such a context if $u \equiv u(t, x)$ is a spatially $\mathbb{T}^{N}$-periodic solution of (1.3), then it becomes a solution of (1.1) with the $\mathbb{T}^{N}$-periodic kernel $K$ defined by

$$
K(x)=\sum_{k \in \mathbb{Z}^{N}} \rho(x+2 \pi k) .
$$

\footnotetext{
* Received by the editors May 23, 2013; accepted for publication (in revised form) January 23, 2014; published electronically May 6, 2014.

http://www.siam.org/journals/sima/46-3/92210.html

${ }^{\dagger}$ CNRS, IMB, UMR 5251, Université de Bordeaux, F-33400 Talence, France (arnaud.ducrot@ubordeaux2.fr, pierre.magal@u-bordeaux2.fr).
} 
The fast decay of $\rho$ is obviously used to ensure the convergence of the above formula.

The problem of form (1.1) or (1.3) describes the spatiotemporal interactions of cells or individuals, whose density at time $t>0$ and located at $x \in \mathbb{R}^{N}$ is denoted $u(t, x)$. The nonlocal flux $\mathbf{J}$, namely, the convolution operator, takes into account the nonlocal spatial interactions between individuals, while the vital dynamics of the population is modeled by the nonlinear function $f$. Throughout this work, function $f: \mathbb{R}^{+} \rightarrow \mathbb{R}$ will be assumed to be of logistic type (see Assumption 1.1 for more precise assumptions), with a prototypical shape $f(u)=u(1-u)$.

The modeling of the motion of cells has a long history. If one omits, for the moment, the vital dynamics of the population, that is, $f(u) \equiv 0$, the nonlocal flux operator $\mathbf{J}$ can be derived from particle interaction using the so-called mean-field or Vlasov limit. This form allows us to take into account long range interaction between cells. Let us now briefly describe such a mean-field approximation. Let us consider $n$ particles in $\mathbb{R}^{N}$ interacting through a potential $V_{n}$. Then the spatial location of each particle is denoted by $X_{k}^{n}(t)$. Then law of motion reads as

$$
\frac{d X_{k}^{n}(t)}{d t}=-\frac{1}{n} \sum_{m=1}^{n} \nabla \rho_{n}\left(X_{k}^{n}(t)-X_{m}^{n}(t)\right), \quad k=1, \ldots, n,
$$

where the potential is assumed to satisfy some scaling property:

$$
\rho_{n}(x)=n^{\beta} \rho\left(n^{\frac{\beta}{N}} x\right)
$$

for some $\beta \in[0,1]$ and where $\rho$ is a fixed given potential. Using the above particle interaction modeling, the corresponding macroscopic law of motion is obtained by investigating the convergence $n \rightarrow \infty$ of the empirical measure $\mu_{n}(t)$ defined by

$$
\mu_{n}(t)=\frac{1}{n} \sum_{k=1}^{n} \delta_{X_{k}^{n}(t)} .
$$

Using this framework, the scaling with $\beta=0$ (resp., $\beta \in(0,1)$, resp. $\beta=1$ ) corresponds to the so-called Vlasov limit (resp., mesoscale limit, resp., hydrodynamics limit) (see $[24,22]$ ). Roughly speaking, in one space dimension, that is, $N=1, n^{-1}$ corresponds to the characteristic length between particles, while $n^{-\beta}$ corresponds to the characteristic length of interaction induced by the potential $\rho_{n}$. The Vlasov framework, namely, $\beta=0$, corresponds to order 1 length of interaction, that is, long range interactions.

All these rescaled limits have been rigorously and formally investigated in the literature not only for deterministic system (1.5) but also for noisy perturbation of such systems. Let us mention the work of Oelschläger [23], where the author derived the flux formulation $\mathbf{J}$ from the noisy system (1.5) with (1.6) and $\beta=0$. In this work the author also considers the case $\beta \in(0,1)$ and derived Darcy's law for moderately interacting potential (fast decay). In [24] the same author investigated the limit behavior $n \rightarrow \infty$ of (1.5) (without noise perturbation) for the case $\beta \in(0,1)$ as well as the one-dimensional hydrodynamic limit. We also refer the reader to Morale, Capasso, and Oelschläger [22] and Bodnar and Velazquez [9] for more modeling details and formal derivations. Let us finally refer to Capasso and Morale [12] for rigorous derivation of macroscopic equation coupling several scales and stochastic effects.

Note that the nonlocal law of motion described by (1.3) posed on $\mathbb{R}^{N}$ with $f(u) \equiv$ 0 arises in many applicative fields and has attracted the attention of many researchers. 
The equation takes the form

$$
\partial_{t} u(t, x)=\nabla \cdot(u(t, x) \nabla[\rho * u(t, .)](x)) .
$$

We refer the reader to the introduction of the recent paper of Bertozzi, Garnett, and Laurent [5] for the list of applications for different fields. This class of systems has been recently studied in $[5,10]$ and $[26]$ with an additional heterogeneous transport term (see also the references therein). Further results in $[2,3,4,5,11,14]$ have been obtained for (1.7) with a linear or nonlinear diffusive perturbation.

In the context of population dynamics, a nonlocal equation similar to (1.1) with diffusive perturbation was previously considered in [21]. We also refer the reader to Leverentz, Topaz, and Bernoff [19] and the references therein for swarming models and numerical experiments. In the context of cell-cell adhesion, several works based on the article of Armstrong, Painter, and Sherratt [1] consider some nonregular kernel $K$ (see also [6, 7] for more results on this topic). Let us also mention that system (1.1) or (1.3) is also closely related to the so-called hyperbolic Keller-Segel equation. Indeed the hyperbolic Keller-Segel equation with linear sensitivity and a logistic perturbation takes the following form (see [25] for more results on this subject):

$$
\left\{\begin{array}{l}
\partial_{t} u(t, x)=\nabla \cdot[\chi u(t, x) \nabla v(t, x)]+f(u) \text { for } t>0 \text { and } x \in \mathbb{R}^{N}, \\
\left(1-\varepsilon^{2} \Delta\right) v(t, x)=u(t, x) \\
u(0, .)=u_{0} \in L_{+}^{\infty}\left(\mathbb{R}^{N}\right) .
\end{array}\right.
$$

Here $\chi \in \mathbb{R}^{*}$ denotes the sensitivity parameter. It is negative when the substance with concentration denoted by $v$ is attractive, while $\chi>0$ if it has a chemorepulsive effect. Next recall that

$$
\left(I-\varepsilon^{2} \Delta\right)^{-1}(\varphi)(x)=\int_{0}^{\infty} e^{-l} T_{\varepsilon^{2} \Delta}(l)(\varphi)(x) d l
$$

and

$$
T_{\varepsilon^{2} \Delta}(t)(\varphi)(x)=\frac{1}{\left(4 \pi \varepsilon^{2} t\right)^{\frac{N}{2}}} \int_{\mathbb{R}^{N}} \varphi(x-y) e^{-\frac{\|y\|^{2}}{4 \pi \varepsilon^{2} t}} d y .
$$

It follows that

$$
v-\varepsilon^{2} \Delta v=u \Leftrightarrow v(t, x)=\int_{\mathbb{R}^{N}} \rho_{N}(x-y) u(t, y) d y,
$$

where

$$
\rho_{N}(x)=\int_{0}^{\infty} \frac{e^{-l}}{\left(4 \pi \varepsilon^{2} l\right)^{\frac{N}{2}}} e^{-\frac{\|x\|^{2}}{4 \pi \varepsilon^{2} l}} d l .
$$

In particular for $N=1$ we have

$$
\rho_{1}(x):=\frac{\varepsilon^{-1}}{2} e^{-\varepsilon^{-1}|x|} .
$$

Hence the hyperbolic Keller-Segel equation is rewritten as (1.1) with the kernel $\rho \equiv$ $\chi \rho_{N}$. For this class of systems the kernel $K$ defined in (1.4) has a singularity only at $x=0$. Such a situation will not enter the framework of this work. 
Coming back to (1.1) the assumptions of this work read as follows.

Assumption 1.1. Function $f: \mathbb{R}^{+} \rightarrow \mathbb{R}$ is concave and takes the form of

$$
f(u)=u h(u) \quad \forall u \geq 0
$$

for some function $h: \mathbb{R}^{+} \rightarrow \mathbb{R}$ of the class $C^{1}$ that satisfies $h(1)=0$ and the following changed sign condition:

$$
h(u)>0 \forall u \in[0,1), \quad h(u)<0 \forall u>1, \text { and } \lim _{u \rightarrow \infty} h(u)=-\infty .
$$

Note that such an assumption holds true for the prototypical function $h(u) \equiv 1-u$. Our second assumption is related to the properties of the kernel operator $K$.

Assumption 1.2. Function $K: \mathbb{R}^{N} \rightarrow \mathbb{R}$ satisfies the following properties:

(i) $K$ is a $\mathbb{T}^{N}$-periodic function of the class $C^{3}$ on $\mathbb{R}^{N}$.

(ii) The Fourier's coefficients of function $K$ on $\mathbb{T}^{N}$ denoted by $\left\{c_{n}[K]\right\}_{n \in \mathbb{Z}^{N}}$ satisfy $c_{n}[K]>0$ for all $n \in \mathbb{Z}^{N} \backslash\{0\}$. Here the Fourier coefficients are defined by

$$
c_{n}[K]=\left|\mathbb{T}^{N}\right|^{-1} \int_{\mathbb{T}^{N}} e^{-i n x} K(x) d x \quad \forall n \in \mathbb{Z}^{N} .
$$

Note that condition (ii) in the above assumption is related to a repulsive property of the kernel, so that (1.1) exhibits a diffusion-like effect. This will be used in a forthcoming modeling work on cell motion [17] (see also [18] for more results on this subject).

If we come back to the formulation (1.3) with kernel $\rho$, let us note that Assumption 1.2 (ii) can be rewritten as follows in terms of Fourier transform of $\rho$ using the relationship (1.4):

$$
c_{n}[K]=\left|\mathbb{T}^{N}\right|^{-1} \hat{\rho}\left(\frac{n}{2 \pi}\right)>0 \quad \forall n \in \mathbb{Z}^{N} \backslash\{0\},
$$

where $\widehat{\rho}$ denotes the Fourier transform of $\rho$ defined by

$$
\widehat{\rho}(\xi):=\int_{\mathbb{R}^{N}} \rho(x) e^{-2 \pi i x \cdot \xi} d x \quad \text { for } \xi \in \mathbb{R}^{\mathbb{N}} .
$$

Due to this remark, if $\rho: \mathbb{R}^{N} \rightarrow \mathbb{R}$ satisfies $\rho \in W^{3,1}\left(\mathbb{R}^{N}\right) \cap C^{3}\left(\mathbb{R}^{N}\right)$, for each multi-index $\alpha$ of length $|\alpha| \leq 3$ the series

$$
x \mapsto \sum_{k \in \mathbb{Z}^{N}} D^{\alpha} \rho(x+2 \pi k)
$$

is uniformly converging on $[-\pi, \pi]^{N}$, and $\widehat{\rho}(\xi)>0$ for all $\xi \in \mathbb{R}^{N}$, then function $K$ defined by (1.4) satisfies Assumption 1.2.

Example 1.3. Assuming that $N=1$, it is readily checked that the kernel

$$
\rho(x)=e^{-x^{2}}\left(\text { resp., } \rho(x)=\frac{2}{1+4 \pi^{2} x^{2}}\right)
$$

satisfies all the aforementioned assumptions and its Fourier transform is

$$
\widehat{\rho}(\xi)=\sqrt{\pi} e^{-(\pi \xi)^{2}}\left(\text { resp., } \widehat{\rho}(\xi)=e^{-|\xi|}\right) .
$$


Of course the kernel corresponding to the one-dimensional hyperbolic Keller-Segel equation $\rho(x)=e^{-|x|}$ also has a positive Fourier transform. But as mentioned above, due the singularity of $\rho$ at 0 , we will not consider this class of problems.

Example 1.4. As mentioned above, the regularity assumption for $\rho$ is not satisfied for the one-dimensional hyperbolic Keller-Segel equation. However, the assumptions considered in this work allow us to deal with the following modified version of the hyperbolic Keller-Segel equation with chemorepulsive effect:

$$
\left\{\begin{array}{l}
\partial_{t} u(t, x)=\partial_{x}\left(u(t, x) \partial_{x} v(t, x)\right)+f(u(t, x)) \text { for } t \geq 0 \text { and } x \in \mathbb{R} \\
\left(1-\varepsilon^{2} \partial_{x}^{2}\right)^{k} v(t, x)=u(t, x), \\
u(0, .)=u_{0} \in L_{+}^{\infty}(\mathbb{R})
\end{array}\right.
$$

with $k \geq 2$. Indeed, in that case one has $v=\left(\rho_{1}^{* k} * u\right)$ with $\rho_{1}(x):=\frac{\varepsilon^{-1}}{2} e^{-\varepsilon^{-1}|x|}$. For $k \geq 2$ we have $\rho_{1}^{* k} \in W^{2 k-1,1}(\mathbb{R}) \cap C^{2 k-2}(\mathbb{R})$. Moreover the Fourier transform is positive since $\widehat{\rho_{1}^{* k}}(\xi)=\widehat{\rho}_{1}(\xi)^{k}>0$. Hence we deduce that Assumption 1.1 is satisfied for $k \geq 3$.

Assumption 1.2 is a relatively strong assumption on the interaction kernel. This type of condition has been used by Chayes and Panferov [14, Corollary 2.7] to prove a convergence result for similar system with a linear diffusive perturbation and no logistic term. Bernoff and Topaz [2] discuss the positivity condition on the Fourier transform of the interaction kernel for this class of model problems (without the logistic term). As far as we know, the logistic effect together with nonlocal diffusive motion (namely, (1.1)) has not yet been considered in the literature.

2. Preliminary. The aim of this preliminary is to derive the existence and uniqueness of solution for (1.1)-(1.2) and to provide estimates of the solutions that will be used in what follows to derive their asymptotic behavior.

Before going further let us introduce some notation that will be used in the paper.

For each $k \in \mathbb{N}$, let us denote by $C_{\sharp}^{k}\left(\mathbb{R}^{N}\right)$ the Banach space of functions of class $C^{k}$ from $\mathbb{R}^{N}$ into $\mathbb{R}$ and $[0,2 \pi]^{N}$-periodic endowed with the usual sup-norm

$$
\|\varphi\|_{k, \infty}=\sum_{p=0}^{k} \sup _{x \in \mathbb{R}^{N}}\left|D^{p} \varphi(x)\right|
$$

For each $p \in[1,+\infty]$, let us denote by $L_{\sharp}^{p}\left(\mathbb{R}^{N}\right)$ the space of measurable and $[0,2 \pi]^{N}$ periodic functions from $\mathbb{R}^{N}$ to $\mathbb{R}$ such that

$$
\|\varphi\|_{L_{\sharp}^{p}\left(\mathbb{R}^{N}\right)}:=\|\varphi\|_{L^{p}\left((-\pi, \pi)^{N}\right)}<+\infty .
$$

Then $L_{\sharp}^{p}\left(\mathbb{R}^{N}\right)$ endowed with the norm $\|\varphi\|_{L_{\sharp}^{p}\left(\mathbb{R}^{N}\right)}$ is a Banach space. We also introduce its positive cone $L_{\sharp,+}^{p}\left(\mathbb{R}^{N}\right)$ consisting of all functions in $L_{\sharp}^{p}\left(\mathbb{R}^{N}\right)$ almost everywhere positive.

Finally if $(X, d)$ is a metric space, then $\operatorname{Lip}(X)$ denotes the Banach space of bounded and Lipschitz continuous functions from $X$ into $\mathbb{R}$ endowed with the usual Lipschitz norm

$$
\|f\|_{\text {Lip }}=\sup _{x \in X}|f(x)|+\sup _{(x, y) \in X^{2}, x \neq y} \frac{|f(x)-f(y)|}{d(x, y)} \forall f \in \operatorname{Lip}(X) .
$$


2.1. Solution integrated along the characteristics. In this section we investigate the existence of solution of (1.1) with initial data $u_{0} \in L_{\sharp}^{\infty}\left(\mathbb{R}^{N}\right)$. To do so let us first investigate the characteristic curves of the problem.

Lemma 2.1. Let Assumption 1.2 (i) be satisfied. Let $u \in C\left([0, \tau], L_{\sharp}^{1}\left(\mathbb{R}^{N}\right)\right)$. Then the map $\mathbf{v}(t, x)=(\nabla K \circ u(t,)).(x)$ belongs to $C\left([0, \tau], C_{\sharp}^{1}\left(\mathbb{R}^{N}\right)\right)^{N}$ and satisfies the following estimates:

$$
\begin{aligned}
& \|\mathbf{v}(t, .)\|_{0, \infty} \leq\|\nabla K\|_{0, \infty}\|u(t, .)\|_{L_{\sharp}^{1}\left(\mathbb{R}^{N}\right)}, \\
& \|\operatorname{div} \mathbf{v}(t, .)\|_{0, \infty} \leq\|\Delta K\|_{0, \infty}\|u(t, .)\|_{L_{\sharp}^{1}\left(\mathbb{R}^{N}\right) .} .
\end{aligned}
$$

The proof of this lemma is omitted. Next the following lemma will allow us to define the characteristic curves.

Lemma 2.2. Let Assumption 1.2 (i) be satisfied. Let $u \in C\left([0, \tau], L_{\sharp}^{1}\left(\mathbb{R}^{N}\right)\right)$ be given. Then setting for $\mathbf{v}(t, x)=(\nabla K \circ u(t,)).(x)$ the nonautonomous system

$$
\left\{\begin{array}{l}
\partial_{t} \Pi_{\mathbf{v}}(t, s ; x)=-\mathbf{v}\left(t, \Pi_{\mathbf{v}}(t, s ; x)\right) \text { for each } t \in[0, \tau] \\
\Pi_{\mathbf{v}}(s, s ; x)=x
\end{array}\right.
$$

posed for each $s \in[0, \tau]$ and each $x \in \mathbb{R}^{N}$ generates a unique nonautonomous continuous flow $\left\{\Pi_{\mathbf{v}}(t, s)\right\}_{t, s \in[0, \tau]}$, that is to say,

$$
\Pi_{\mathbf{v}}(t, r ; x) \Pi_{\mathbf{v}}(r, s ; x)=\Pi_{\mathbf{v}}(t, s ; x) \quad \forall t, s, r \in[0, \tau] \text { and } \Pi_{\mathbf{v}}(s, s ; .)=I,
$$

and the map $(t, s, x) \rightarrow \Pi_{\mathbf{v}}(t, s ; x)$ is continuous. Moreover for each $t, s \in[0, \tau]$, we have

$$
\Pi_{\mathbf{v}}(t, s ; x+2 \pi k)=\Pi_{\mathbf{v}}(t, s ; x)+2 \pi k \quad \forall x \in \mathbb{R}^{\mathbb{N}}, k \in \mathbb{Z}^{N},
$$

the map $x \rightarrow \Pi_{\mathbf{v}}(t, s ; x)$ is continuously differentiable, and one has

$$
\operatorname{det}\left(\partial_{x} \Pi_{\mathbf{v}}(t, s ; x)\right)=\exp \left(-\int_{s}^{t} \operatorname{div} \mathbf{v}\left(l, \Pi_{\mathbf{v}}(l, s ; x)\right) d l\right) .
$$

Proof. This result follows by using classical arguments on ordinary differential equations and the estimations obtained in Lemma 2.1.

In order to make the notion of solution we will use in this work precise, assume first that $u \in C^{1}([0, \tau] \times \mathbb{R}, \mathbb{R}) \cap C\left([0, \tau], C_{\sharp,+}^{0}\left(\mathbb{R}^{N}\right)\right)$ is a classical solution of (1.1)(1.2). By setting for $0 \leq s \leq t \leq \tau$

$$
V_{\mathbf{v}}(t, s ; x)=\exp \left(-\int_{s}^{t} \operatorname{div} \mathbf{v}\left(l, \Pi_{\mathbf{v}}(l, s ; x)\right) d l\right),
$$

then one has

$$
\begin{aligned}
\frac{d}{d t}\left(u\left(t, \Pi_{\mathbf{v}}(t, 0) x\right) V_{\mathbf{v}}(t, 0 ; x)\right)= & {\left[\partial_{t} u\left(t, \Pi_{\mathbf{v}}(t, 0 ; x)\right)-\mathbf{v}\left(t, \Pi_{\mathbf{v}}(t, 0 ; x) \nabla u\left(t, \Pi_{\mathbf{v}}(t, 0 ; x)\right)\right.\right.} \\
& -u\left(t, \Pi_{\mathbf{v}}(t, 0 ; x) \operatorname{div} \mathbf{v}\left(t, \Pi_{\mathbf{v}}(t, 0 ; x)\right)\right] V_{\mathbf{v}}(t, 0 ; x) .
\end{aligned}
$$

Hence a classical solution of (1.1)-(1.2) satisfies

$$
\frac{d}{d t}\left(u\left(t, \Pi_{\mathbf{v}}(t, 0 ; x)\right) V_{\mathbf{v}}(t, 0 ; x)\right)=h\left(u\left(t, \Pi_{\mathbf{v}}(t, 0 ; x)\right)\right) u\left(t, \Pi_{\mathbf{v}}(t, 0 ; x)\right) V_{\mathbf{v}}(t, 0 ; x) .
$$


It follows by using (2.3) that

$$
u\left(t, \Pi_{\mathbf{v}}(t, 0 ; x)\right)=\exp \left(\int_{0}^{t} h\left(u\left(l, \Pi_{\mathbf{v}}(l, 0 ; x)\right)\right)+\operatorname{div} \mathbf{v}\left(l, \Pi_{\mathbf{v}}(l, 0 ; x)\right) d l\right) u_{0}(x),
$$

or equivalently

$$
u(t, x)=\exp \left(\int_{0}^{t} h\left(u\left(l, \Pi_{\mathbf{v}}(l, t ; x)\right)\right)+\operatorname{div} \mathbf{v}\left(l, \Pi_{\mathbf{v}}(l, t ; x)\right) d l\right) u_{0}\left(\Pi_{\mathbf{v}}(0, t ; x)\right) .
$$

Set

$$
\mathbf{v}(t, x):=\int_{\mathbb{T}^{N}} \nabla K(x-z) u(t, z) d z .
$$

By using the change of variable $\widehat{z}=\Pi_{\mathbf{v}}(0, t ; z)$ we obtain

$$
\mathbf{v}(t, x):=\int_{\mathbb{T}^{N}} \nabla K\left(x-\Pi_{\mathbf{v}}(t, 0 ; \widehat{z})\right) u\left(t, \Pi_{\mathbf{v}}(t, 0 ; \widehat{z})\right) \exp \left(-\int_{0}^{t} \operatorname{div} \mathbf{v}\left(l, \Pi_{\mathbf{v}}(l, 0 ; \widehat{z})\right) d l\right) d \widehat{z} .
$$

Combining the above formula together with (2.4), we obtain

$$
\mathbf{v}(t, x)=\int_{\mathbb{T}^{N}} \nabla K\left(x-\Pi_{\mathbf{v}}(t, 0 ; \widehat{z})\right) e^{\int_{0}^{t} h\left(u\left(l, \Pi_{\mathbf{v}}(l, 0 ; \widehat{z})\right)\right) d l} u_{0}(\widehat{z}) d \widehat{z} .
$$

The above computations lead us to the following definition of solution.

Definition 2.3 (solution integrated along the characteristics). Let $u_{0} \in L_{\sharp,+}^{\infty}\left(\mathbb{R}^{N}\right)$ be given. Let $\tau>0$ be given. A function $u \in C\left([0, \tau], L_{\sharp,+}^{1}\left(\mathbb{R}^{N}\right)\right) \cap L^{\infty}\left((0, \tau), L_{\sharp,+}^{\infty}\left(\mathbb{R}^{N}\right)\right)$ is said to be a solution integrated along the characteristics of (1.1)-(1.2) if $u$ satisfies (2.5) with $\mathbf{v}$ defined in (2.6).

Then our existence result reads as follows.

Theorem 2.4. Let Assumptions 1.1 and 1.2 (i) be satisfied. For each $u_{0} \in$ $L_{\sharp,+}^{\infty}\left(\mathbb{R}^{N}\right)$, system $(1.1)-(1.2)$ has a unique solution integrated along the characteristics $t \rightarrow U(t) u_{0}$ in $C\left([0,+\infty), L_{\sharp,+}^{1}\left(\mathbb{R}^{N}\right)\right) \cap L_{\text {loc }}^{\infty}\left([0, \infty), L_{\sharp,+}^{\infty}\left(\mathbb{R}^{N}\right)\right)$. Moreover $\{U(t)\}_{t \geq 0}$ is a continuous semiflow on $L_{\sharp,+}^{\infty}\left(\mathbb{R}^{N}\right)$, that is to say,

(i) $U(t) U(s)=U(t+s)$ for all $t, s \geq 0$ and $U(0)=I$;

(ii) the map $\left(t, u_{0}\right) \rightarrow U(t) u_{0}$ maps every bounded set of $[0,+\infty) \times L_{\sharp,+}^{\infty}(\mathbb{R})$ into a bounded set of $L_{\sharp,+}^{\infty}(\mathbb{R})$;

(iii) (Continuity) if $\left\{t_{n}\right\}_{n \in \mathbb{N}}(\subset[0,+\infty)) \rightarrow t<+\infty$ and $\left\{u_{0}^{n}\right\}_{n \in \mathbb{N}}$ is a bounded sequence in $L_{\sharp,+}^{\infty}\left(\mathbb{R}^{N}\right)$ such that $\left\|u_{0}^{n}-u_{0}\right\|_{L_{\sharp}^{1}\left(\mathbb{R}^{N}\right)} \rightarrow 0$ as $n \rightarrow+\infty$, then

$$
\left\|U\left(t_{n}\right) u_{0}^{n}-U(t) u_{0}\right\|_{L_{\sharp}^{1}\left(\mathbb{R}^{N}\right)} \rightarrow 0 \text { as } n \rightarrow+\infty .
$$

The semiflow $U$ also satisfies the two following properties:

$$
\begin{gathered}
U(t) u_{0} \geq 0 \quad \forall u_{0} \geq 0, \forall t \geq 0, \\
\left\|U(t) u_{0}\right\|_{L_{\sharp}^{1}\left(\mathbb{R}^{N}\right)} \leq e^{M t}\left\|u_{0}\right\|_{L_{\sharp}^{1}\left(\mathbb{R}^{N}\right)} \quad \forall t \geq 0 \text { with } M=\sup _{u \geq 0} h(u) .
\end{gathered}
$$


Furthermore, if in addition $u_{0} \in W_{\sharp}^{1,1}\left(\mathbb{R}^{N}\right)$, then $U(.) u_{0} \in C^{1}\left([0,+\infty), L_{\sharp}^{1}\left(\mathbb{R}^{N}\right)\right)$, and if in addition $u_{0} \in C_{\sharp}^{1}\left(\mathbb{R}^{N}\right)$, then $u(t, x)=U(t)\left(u_{0}\right)(x)$ belongs to $C^{1}([0,+\infty) \times$ $\left.\mathbb{R}^{N}, \mathbb{R}\right)$ and $u(t, x)$ is a classical solution of system (1.1)-(1.2).

In order to prove the above lemma we set

$$
w(t, x):=u\left(t, \Pi_{\mathbf{v}}(t, 0 ; x)\right) .
$$

Next (2.4) yields to

$$
w(t, x)=\exp \left(\int_{0}^{t} h(w(l, x))+\operatorname{div} \mathbf{v}\left(l, \Pi_{\mathbf{v}}(l, 0 ; x)\right) d l\right) u_{0}(x) .
$$

Hence one obtains

$$
w \in C\left([0, \tau], L_{\sharp,+}^{\infty}\left(\mathbb{R}^{N}\right)\right) .
$$

On the other hand, note that (2.7) provides

$$
\mathbf{v}(t, x):=\int_{\mathbb{T}^{N}} \nabla K\left(x-\Pi_{\mathbf{v}}(t, 0 ; \widehat{z})\right) e^{\int_{0}^{t} h(w(l, \widehat{z})) d l} u_{0}(\widehat{z}) d \widehat{z} .
$$

Then (2.11)-(2.12) leads us to the following fixed point problem:

$$
\left(\begin{array}{c}
w \\
\mathbf{v}
\end{array}\right) \in C\left([0, \tau], L_{\sharp,+}^{\infty}\left(\mathbb{R}^{N}\right)\right) \times C\left([0, \tau], C_{\sharp}^{1}\left(\mathbb{R}^{N}\right)^{N}\right) \text { and }\left(\begin{array}{l}
w \\
\mathbf{v}
\end{array}\right)=\mathcal{T}\left(\begin{array}{l}
w \\
\mathbf{v}
\end{array}\right),
$$

wherein the operator $\mathcal{T}$ is defined by $\mathcal{T}\left(\begin{array}{c}w \\ \mathbf{v}\end{array}\right)=\left(\begin{array}{c}w^{\prime} \\ \mathbf{v}^{\prime}\end{array}\right)$ and

$$
\begin{aligned}
& w^{\prime}(t, x)=\exp \left(\int_{0}^{t} h(w(l, x))+\operatorname{div} \mathbf{v}^{\prime}\left(l, \Pi_{\mathbf{v}}(l, 0 ; x)\right) d l\right) u_{0}(x), \\
& \mathbf{v}^{\prime}(t, x)=\int_{\mathbb{T}^{N}} \nabla K\left(x-\Pi_{\mathbf{v}}(t, 0 ; \widehat{z})\right) e^{\int_{0}^{t} h(w(l, \widehat{z})) d l} u_{0}(\widehat{z}) d \widehat{z} .
\end{aligned}
$$

We will now sketch the proof of Theorem 2.4 by showing that the contraction mapping theorem applies for $\mathcal{T}$ as soon as $\tau>0$ is small enough. This will ensure the existence and uniqueness of a local solution. To do so we fix $\tau>0$, a value that will be chosen later on, and we consider the Banach space $X$ defined by

$$
X=C\left([0, \tau], L_{\sharp}^{\infty}\left(\mathbb{R}^{N}\right)\right) \times C\left([0, \tau], C_{\sharp}^{1}\left(\mathbb{R}^{N}\right)^{N}\right),
$$

endowed with the usual product norm:

$$
\left\|\left(\begin{array}{c}
w \\
\mathbf{v}
\end{array}\right)\right\|_{X}=\|w\|_{C\left([0, \tau], L_{\sharp}^{\infty}\left(\mathbb{R}^{N}\right)\right)}+\|\mathbf{v}\|_{C\left([0, \tau], C_{\sharp}^{1}\left(\mathbb{R}^{N}\right)^{N}\right)} .
$$

We also introduce the closed subset $X^{+} \subset X$ defined by

$$
X^{+}=C\left([0, \tau], L_{\sharp,+}^{\infty}\left(\mathbb{R}^{N}\right)\right) \times C\left([0, \tau], C_{\sharp}^{1}\left(\mathbb{R}^{N}\right)^{N}\right) .
$$

Now we consider the operator $\mathcal{T}: X^{+} \rightarrow X$ defined by $\mathcal{T}\left(\begin{array}{c}w \\ \mathbf{v}\end{array}\right)=\left(\begin{array}{c}w^{\prime} \\ \mathbf{v}^{\prime}\end{array}\right)$, with $\left(w^{\prime}, \mathbf{v}\right)$ defined in (2.13). Note that due to (2.5) one has

$$
\mathcal{T}\left(X^{+}\right) \subset X^{+} .
$$


Next let us set

$$
U_{0}(t) \equiv\left(\begin{array}{c}
u_{0} \\
\mathbf{v}_{\mathbf{0}}
\end{array}\right) \in X^{+} \text {with } \mathbf{v}_{\mathbf{0}}=\nabla K \circ u_{0}
$$

For each $U \in X$ and $\kappa>0$ we denote by $\bar{B}_{X}(U, \kappa)$ the closed ball in $X$ of center $U$ and radius $\kappa$.

Now let $\kappa>0$ be given. We claim that there exists $\widehat{\tau}>0$ such that for each $\tau \in(0, \widehat{\tau})$

$$
\mathcal{T}\left(X^{+} \cap \bar{B}_{X}\left(U_{0}, \kappa\right)\right) \subset X^{+} \cap \bar{B}_{X}\left(U_{0}, \kappa\right) .
$$

To prove this claim, let $\left(\begin{array}{c}w \\ \mathbf{v}\end{array}\right) \in X^{+} \cap \bar{B}_{X}\left(U_{0}, \kappa\right)$ be given. Note that

$$
\left\|\left(\begin{array}{l}
w \\
\mathbf{v}
\end{array}\right)\right\|_{X} \leq\left\|U_{0}\right\|_{X}+\kappa=: M(\kappa) .
$$

Then recalling the definition of $\left(w^{\prime}, \mathbf{v}^{\prime}\right)$ in $(2.13)$ and setting $H(\kappa)=\sup _{s \in[0, M(\kappa)]} h(s)$ one has

$$
\sup _{t \in[0, \tau]}\left\|\operatorname{div} \mathbf{v}^{\prime}(t, .)\right\|_{L_{\sharp}^{\infty}} \leq\|\Delta K\|_{0, \infty} e^{\tau H(\kappa)}\left\|U_{0}\right\|_{X}=: K_{1}(\tau)
$$

and

$$
\sup _{t \in[0, \tau]}\left\|w^{\prime}(t, .)-u_{0}(.)\right\|_{L_{\sharp}^{\infty}} \leq \tau\left\|U_{0}\right\|_{X}\left(H(\kappa)+K_{1}(\tau)\right) e^{\tau\left(H(\kappa)+K_{1}(\tau)\right)} .
$$

Moreover one has

$$
\sup _{t \in[0, \tau]}\left\|\mathbf{v}^{\prime}(t, .)-\mathbf{v}_{\mathbf{0}}\right\|_{\left(C_{\sharp}^{1}\right)^{N}} \leq 2 \tau\left\|U_{0}\right\|_{X}\|K\|_{3, \infty} e^{\tau H(\kappa)}\left|\mathbb{T}^{N}\right|[M(\kappa)+H(\kappa)] .
$$

Recalling (2.14), the above computation completes the proof of (2.15).

We now claim that there exists $\tau^{*} \in(0, \widehat{\tau})$ such that for each $\tau \in(0, \widehat{\tau})$ there exists $L(\tau) \in(0,1)$ such that for each $\left(\begin{array}{c}w_{1} \\ \mathbf{v}_{1}\end{array}\right),\left(\begin{array}{c}w_{2} \\ \mathbf{v}_{2}\end{array}\right) \in X^{+} \cap \bar{B}_{X}\left(U_{0}, \kappa\right)$

$$
\left\|\mathcal{T}\left(\begin{array}{l}
w_{1} \\
\mathbf{v}_{\mathbf{1}}
\end{array}\right)-\mathcal{T}\left(\begin{array}{l}
w_{2} \\
\mathbf{v}_{\mathbf{2}}
\end{array}\right)\right\|_{X} \leq L(\tau)\left\|\left(\begin{array}{l}
w_{1} \\
\mathbf{v}_{\mathbf{1}}
\end{array}\right)-\left(\begin{array}{l}
w_{2} \\
\mathbf{v}_{\mathbf{2}}
\end{array}\right)\right\|_{X} .
$$

To prove this claim let $\tau \in(0, \widehat{\tau})$ be given. Let $\left(\begin{array}{c}w_{1} \\ \mathbf{v}_{1}\end{array}\right),\left(\begin{array}{c}w_{2} \\ \mathbf{v}_{2}\end{array}\right) \in X^{+} \cap \bar{B}_{X}\left(U_{0}, \kappa\right)$ be given and let $\left(w_{1}^{\prime}, \mathbf{v}_{\mathbf{1}}^{\prime}\right)$ and $\left(w_{2}^{\prime}, \mathbf{v}_{\mathbf{2}}^{\prime}\right)$ be defined as in $(2.13)$ with $(w, \mathbf{v})$ replaced, respectively, by $\left(w_{1}, \mathbf{v}_{\mathbf{1}}\right)$ and $\left(w_{2}, \mathbf{v}_{\mathbf{2}}\right)$. Now before proving $(2.17)$, let us first observe that due to (2.2) and Gronwall's inequality one has for all $t \in[0, \tau]$ and $x \in \mathbb{T}^{N}$

$$
\left.\left\|\Pi_{\mathbf{v}_{\mathbf{1}}}(t, 0, x)-\Pi_{\mathbf{v}_{\mathbf{2}}}(t, 0, x)\right\| \leq \tau\left\|\mathbf{v}_{\mathbf{1}}-\mathbf{v}_{\mathbf{2}}\right\|_{C\left([0, \tau], C_{\sharp}^{1}\right.}\right)^{M(\kappa) \tau} .
$$

From the above estimate we obtain

$$
\begin{aligned}
\left\|\mathbf{v}_{\mathbf{1}}^{\prime}-\mathbf{v}_{\mathbf{2}}^{\prime}\right\| \leq & \|K\|_{3, \infty} \tau\left\|\mathbf{v}_{\mathbf{1}}-\mathbf{v}_{\mathbf{2}}\right\|_{C\left([0, \tau], C_{\sharp}^{1}\right)} e^{M(\kappa) \tau} e^{\tau H(\kappa)}\left|\mathbb{T}^{N}\right|\left\|u_{0}\right\|_{\infty} \\
& +\|K\|_{2, \infty} e^{\tau H(\kappa)} \tau H^{\prime}(\kappa)\left\|w_{1}-w_{2}\right\|_{C\left([0, \tau], L_{\sharp}^{\infty}\right)}\left|\mathbb{T}^{N}\right|\left\|u_{0}\right\|_{\infty} .
\end{aligned}
$$

Here we have set

$$
H^{\prime}(\kappa)=\sup _{s \in[0, M(\kappa)]}\left|h^{\prime}(s)\right|
$$


Recalling (2.16), one gets for each $t \in[0, \tau]$

$$
\begin{aligned}
\left\|w_{1}^{\prime}(t, .)-w_{2}^{\prime}(t, .)\right\|_{L_{\sharp}^{\infty}} \leq \tau\left\|U_{0}\right\|_{X} e^{\tau H(\kappa)+\tau K_{1}(\tau)}\left\{H^{\prime}(\kappa)\left\|w_{1}-w_{2}\right\|_{C\left([0, \tau], L_{\sharp}^{\infty}\right)}\right. \\
\left.+\sup _{l \in[0, \tau]}\left\|\operatorname{div} \mathbf{v}_{\mathbf{1}}^{\prime}\left(l, \Pi_{\mathbf{v}_{\mathbf{1}}}(l, 0 ; .)\right)-\operatorname{div} \mathbf{v}_{\mathbf{2}}^{\prime}\left(l, \Pi_{\mathbf{v}_{\mathbf{2}}}(l, 0 ; .)\right)\right\|_{L_{\sharp}^{\infty}} d l\right\} .
\end{aligned}
$$

To complete the proof of (2.17) let us notice that for each $l \in[0, \tau]$ one has

$$
\begin{aligned}
\| \operatorname{div} \mathbf{v}_{\mathbf{1}}^{\prime}\left(l, \Pi_{\mathbf{v}_{\mathbf{1}}}(l, 0 ; .)\right)- & \operatorname{div} \mathbf{v}_{\mathbf{2}}^{\prime}\left(l, \Pi_{\mathbf{v}_{\mathbf{2}}}(l, 0 ; .)\right) \|_{L_{\sharp}^{\infty}} \\
& \leq 2\left\|U_{0}\right\|_{X} e^{\tau H(\kappa)}\|K\|_{3, \infty} \sup _{x \in \mathbb{T}^{N}}\left\|\Pi_{\mathbf{v}_{\mathbf{1}}}(l, 0, x)-\Pi_{\mathbf{v}_{\mathbf{2}}}(l, 0, x)\right\| \\
& +\left\|U_{0}\right\|_{X}\|K\|_{2, \infty} e^{\tau H(\kappa)} H^{\prime}(\kappa) \tau\left\|w_{1}-w_{2}\right\|_{C\left([0, \tau], L_{\sharp}^{\infty}\right)} .
\end{aligned}
$$

Hence (2.17) follows from (2.19)-(2.20) making use of (2.18).

Finally one concludes from (2.15) and (2.17) that for $\tau$ small enough, the contraction mapping theorem applies to operator $\mathcal{T}$. Hence the operator $\mathcal{T}$ has a unique fixed point in $X^{+} \cap \bar{B}_{X}\left(U_{0}, \kappa\right)$. Recalling (2.10), this ensures the existence and uniqueness of the local solution integrated along the characteristic of (1.1) as well as (2.8).

Let us mention that the smoothness of the kernel $K$ (namely, of the class $C^{3}$ ) is a key ingredient in deriving the existence and the uniqueness of the solution. We refer the reader to [6, 7] for other methods whenever $K$ is less smooth (and without logistic nonlinearity). See also [20] for other existence and uniqueness results for the two-dimensional Euler equation in fluid mechanics.

It remains to show that the semiflow is globally defined. Recalling the definition of $M$ in (2.9), one deduces from (2.5) that

$$
u(t, x) \leq \exp (M t) V_{\mathbf{v}}\left(t, 0 ; \Pi_{\mathbf{v}}(0, t ; x)^{-1}\right) u_{0}\left(\Pi_{\mathbf{v}}(0, t ; x)\right),
$$

which completes the proof (2.9). Recalling Lemma 2.1 and $u_{0} \in L_{\sharp}^{\infty}\left(\mathbb{R}^{N}\right)$, one obtains that there exists some constant $\widehat{M}>0$ such that

$$
\|\mathbf{v}(t, .)\|_{0, \infty}+\|\operatorname{div} \mathbf{v}(t, .)\|_{0, \infty} \leq \widehat{M} \exp (M t) .
$$

Recalling that

$$
V_{\mathbf{v}}\left(t, 0 ; \Pi_{\mathbf{v}}(0, t ; x)^{-1}\right)=\exp \left(\int_{0}^{t} \operatorname{div} \mathbf{v}\left(l, \Pi_{v}(l, 0) x\right) d l\right),
$$

one infers from $(2.21)$ and $(2.22)$ that there exists some constant $\widetilde{M}>0$ such that

$$
\|u(t, .)\|_{0, \infty} \leq \exp (M t) \exp \left(\frac{\widehat{M}}{M} \exp (M t)\right)\left\|u_{0}\right\|_{0, \infty} \forall t \geq 0 .
$$

The result follows.

Remark 2.5 (conservation law). The above computations lead us to the following conservation law: For each Borel set $A \subset \mathbb{T}^{N}$ and each $0 \leq s \leq t$,

$$
\int_{\Pi_{\mathbf{v}}(t, s ; .) A} u(t, x) d x=\int_{A} \exp \left[\int_{s}^{t} h\left(u\left(l, \Pi_{\mathbf{v}}(l, s ; z)\right)\right)\right] u(s, z) d z .
$$


3. A priori estimates and energy functional. When dealing with (1.3) for $f \equiv 0$ and with initial datum in $L^{1}\left(\mathbb{R}^{N}\right)$, it is well known (see, for instance, $[11,26]$ ) that the functional $\mathcal{E}[u(t,)]:.=\int_{\mathbb{R}^{N}}(\rho * u(t,)).(x) u(t, x) d x$ is decreasing along the trajectories. In the context of periodic initial datum, one can also check that the functional $\mathcal{E}_{\sharp}$ defined by

$$
\mathcal{E}_{\sharp}[u(t, .)]:=\int_{\mathbb{T}^{N}}(K \circ u(t, .))(x) u(t, x) d x
$$

is also decreasing along the trajectories of (1.1). However, here we shall make use of another energy functional. Let $u \equiv u(t, x)$ be a classical solution of (1.1)-(1.2). Then let us consider the functional

$$
E[u(t, .)]=\frac{1}{\left|T^{N}\right|} \int_{\mathbb{T}^{N}} G(u(t, x)) d x,
$$

wherein function $G:[0, \infty) \rightarrow[0, \infty)$ is defined by

$$
G(u)=u \ln (u)-u+1 .
$$

Then the following lemma holds true.

Lemma 3.1. Let Assumptions 1.1 and 1.2 (i) be satisfied. Let $u_{0} \in L_{\sharp,+}^{\infty}\left(\mathbb{R}^{N}\right)$ be given. Let $u \equiv u(t, x)$ be the solution of (1.1)-(1.2). Then one has for each $(t, \tau) \in(0, \infty)^{2}$ with $t-\tau \geq 0$

$$
\begin{array}{r}
E[u(t+\tau, .)]-E[u(t-\tau, .)]=-\int_{t-\tau}^{t+\tau} \sum_{n \in \mathbb{Z}^{N}}\|n\|^{2} c_{n}(K)\left|c_{n}(u(s, .))\right|^{2} d s \\
-\frac{1}{\left|\mathbb{T}^{N}\right|} \int_{t-\tau}^{t+\tau} \int_{\mathbb{T}^{N}} u(s, x)|\ln (u(s, x))||h(u(s, x))| d x d s .
\end{array}
$$

Proof. Let $u$ be a classical solution of (1.1). Then one has

$$
\frac{d}{d t} E[u(t, .)]=-\frac{1}{\left|T^{N}\right|} \int_{\mathbb{T}^{N}} u|\ln (u)||h(u)| d x+\frac{1}{\left|T^{N}\right|} \int_{\mathbb{T}^{N}} u(\Delta K \circ u) d x .
$$

On the other hand, let us notice that for each $\varphi \in L_{\sharp}^{2}\left(\mathbb{R}^{N}\right)$ one has

$$
\frac{1}{\left|T^{N}\right|} \int_{\mathbb{T}^{N}} \varphi(\Delta K \circ \varphi) d x=\sum_{k \in \mathbb{Z}^{N}} \overline{c_{k}[\varphi]} c_{k}[\Delta K \circ \varphi]=-\sum_{k \in \mathbb{Z}^{N}}\|k\|^{2} c_{k}[K]\left|c_{k}[\varphi]\right|^{2} .
$$

Hence (3.3) holds true for classical solutions.

Let $u_{0} \in L_{\sharp}^{\infty}\left(\mathbb{R}^{N}\right)$ be given and let $u$ denote the corresponding solution of (1.1). Let us consider a sequence $\left\{u_{0}^{n}\right\}_{n \geq 0} \subset C_{\sharp}^{1}\left(\mathbb{R}^{N}\right)$ such that $\left\|u_{0}^{n}-u_{0}\right\|_{L^{1}} \rightarrow 0$ as $n \rightarrow \infty$. If we denote by $u^{n}$ the classical solution of (1.1) with initial data $u_{0}^{n}$, then Theorem 2.4 (iii) ensures that $u^{n} \rightarrow u$ in $C_{l o c}\left([0, \infty) ; L_{\sharp}^{1}\left(\mathbb{R}^{N}\right)\right)$. Since $u^{n}$ satisfies (3.3), $u$ also satisfies (3.3) using the above convergence as well as the Lebesgue convergence theorem. The result follows.

Before using this functional, let us first derive some estimates on the solution of (1.1)-(1.2) provided by Theorem 2.4. Our first estimate reads as follows.

Lemma 3.2. Let Assumptions 1.1 and 1.2 (i) be satisfied. Let $u_{0} \in L_{\sharp,+}^{\infty}\left(\mathbb{R}^{N}\right)$ be given. Then one has

$$
\left\|U(t) u_{0}\right\|_{L_{\sharp}^{1}\left(\mathbb{R}^{N}\right)} \leq \max \left(\left\|u_{0}\right\|_{L_{\sharp}^{1}\left(\mathbb{R}^{N}\right)},\left|\mathbb{T}^{N}\right|\right) \quad \forall t>0 .
$$


Moreover we have the following dissipativity property:

$$
\limsup _{t \rightarrow+\infty}\left\|U(t) u_{0}\right\|_{L_{\sharp}^{1}\left(\mathbb{R}^{N}\right)} \leq\left|\mathbb{T}^{N}\right| .
$$

The above lemma shows the semiflow $U$ is bounded dissipative in $L_{\sharp}^{1}\left(\mathbb{R}^{N}\right)$.

Proof. To prove this estimate we will first deal with the classical solution. To do so let us assume that $u_{0} \in C_{\sharp}^{1}\left(\mathbb{R}^{N}\right)$ with $u_{0} \geq 0$. Let us denote by $u \equiv u(t, x)$ the corresponding classical solution of (1.1) (see Theorem 2.4). Then integrating (1.1) over $\mathbb{T}^{N}$ yields

$$
\frac{d}{d t} \frac{1}{\mid \mathbb{T}^{N \mid}} \int_{\mathbb{T}^{N}} u(t, x) d x=\frac{1}{\left|\mathbb{T}^{N}\right|} \int_{\mathbb{T}^{N}} f(u(t, x)) d x \leq f\left(\frac{1}{\left|\mathbb{T}^{N}\right|} \int_{\mathbb{T}^{N}} u(t, x) d x\right) .
$$

Note that the above inequality holds true using the Jensen inequality (recall that $f$ is assumed to be concave; see Assumption 1.1). The results follows using the usual ordinary differential arguments with Assumption 1.1. The case of solutions integrated along the characteristics (see Definition 2.3) is handled using a regularization sequence of initial data.

Our next estimate reads as follows.

Lemma 3.3. Let Assumptions 1.1 and 1.2 be satisfied. The function

$$
\mathbf{v}(t, x):=(\nabla K \circ u(t, .))(x) \text { satisfies } \mathbf{v} \in L^{\infty}\left((0, \infty), W_{\sharp}^{1, \infty}\left(\mathbb{R}^{N}\right)\right)^{N},
$$

and one has for each $t \geq 0$

$$
\|\operatorname{div} \mathbf{v}(t, .)\|_{L_{\sharp}^{\infty}\left(\mathbb{R}^{N}\right)} \leq\|\Delta K\|_{\infty} \max \left(\left\|u_{0}\right\|_{L_{\sharp}^{1}\left(\mathbb{R}^{N}\right)},\left|\mathbb{T}^{N}\right|\right)
$$

and

$$
\limsup _{t \rightarrow+\infty}\|\operatorname{div} \mathbf{v}(t, .)\|_{\infty} \leq\|\Delta K\|_{\infty}\left|\mathbb{T}^{N}\right| .
$$

The proof of the above lemma is straightforward using Lemma 3.2. It will allow us to derive the following uniform bound.

Lemma 3.4. Let Assumptions 1.1 and 1.2 be satisfied. Then for each initial datum $u_{0} \in L_{\sharp}^{\infty}\left(\mathbb{R}^{N}\right)$, one has for each $t>0$ and a.e. $x \in \mathbb{R}^{N}$

$$
u(t, x) \leq \max \left(\left\|u_{0}\right\|_{\infty}, M_{0}\left(\left\|u_{0}\right\|_{L_{\sharp}^{1}\left(\mathbb{R}^{N}\right)}\right)\right),
$$

where $M_{0}=M_{0}(z)$ is defined using (1.9) by

$$
\begin{aligned}
M_{0}(z) & =\inf \{t>1: C(z)+h(y) \leq 0 \forall y \geq t\} \text { and } \\
C(z) & :=\|\Delta K\|_{0, \infty} \max \left(z,\left|\mathbb{T}^{N}\right|\right) .
\end{aligned}
$$

Moreover we have the following dissipativity property:

$$
\limsup _{t \rightarrow+\infty}\|u(t, .)\|_{0, \infty} \leq M_{0}\left(\left\|u_{0}\right\|_{L_{\sharp}^{1}\left(\mathbb{R}^{N}\right)}\right) .
$$

Proof. Here again we only prove the above estimates for the classical solution. The usual limiting procedure allows us to extend this estimate for solutions integrated along the characteristics according to Definition 2.3. Let $u_{0} \in C_{\sharp}^{1}\left(\mathbb{R}^{N}\right)$ with $u_{0} \geq 0$ 
be given. Let $u \equiv u(t, x)$ be the corresponding classical solution of (1.1). Then one has for each $t>0$ and $x \in \mathbb{T}^{N}$

$$
\partial_{t} u=\mathbf{v} \nabla u+u(\operatorname{div} \mathbf{v}+h(u)) .
$$

On the other hand, recalling the notation of Lemma 2.2, one gets for each $t>0$ and $x \in \mathbb{T}^{N}$

$$
\frac{d u\left(t, \Pi_{\mathbf{v}}(t, 0 ; x)\right)}{d t}=\partial_{t} u\left(t, \Pi_{\mathbf{v}}(t, 0 ; x)\right)-\mathbf{v}\left(t, \Pi_{\mathbf{v}}(t, 0 ; x)\right) \nabla u\left(t, \Pi_{v}(t, 0 ; x)\right) .
$$

Hence by setting $X(t):=u\left(t, \Pi_{\mathbf{v}}(t, 0 ; x)\right)$ one obtains

$$
\frac{d X(t)}{d t}=X(t)\left[\operatorname{div} \mathbf{v}\left(t, \Pi_{v}(t, 0) x\right)+h(X(t))\right] .
$$

Due to Lemma 3.3 we obtain

$$
\frac{d X(t)}{d t} \leq X(t)\left[C\left(\left\|u_{0}\right\|_{L_{\sharp}^{1}\left(\mathbb{R}^{N}\right)}\right)+h(X(t))\right] \quad \text { and } \quad X(0)=u_{0}(x) .
$$

Therefore this yields

$$
u\left(t, \Pi_{v}(t, 0 ; x)\right)=X(t) \leq \max \left(\left\|u_{0}\right\|_{\infty}, M_{0}\left(\left\|u_{0}\right\|_{L_{\sharp}^{1}\left(\mathbb{R}^{N}\right)},\right)\right),
$$

and since the map $x \rightarrow \Pi_{v}(t, 0 ; x)$ is invertible, the proof is complete whenever $u_{0}$ is smooth enough.

Let us prove an identity that will be used in what follows.

Lemma 3.5. Let Assumptions 1.1 and 1.2 be satisfied. Let $u_{0} \in L_{\sharp,+}^{\infty}\left(\mathbb{R}^{N}\right)$ be given and let us denote by $u \equiv u(t, x)$ the solution of $(1.1)-(1.2)$. For each $F \in C^{1}\left(\mathbb{T}^{N} \times \mathbb{R}\right)$ the map $t \mapsto \int_{\mathbb{T}^{N}} F(x, u) d x$ is of the class $C^{1}$ and one has

$$
\begin{aligned}
\frac{d}{d t} \int_{\mathbb{T}^{N}} F(x, u(t, x)) d x= & \int_{\mathbb{T}^{N}}\left[u(t, x) F_{u}(x, u(t, x))-F(x, u(t, x))\right] \operatorname{div} \mathbf{v}(t, x) d x \\
& -\int_{\mathbb{T}^{N}} \nabla_{x} F(x, u(t, x)) \mathbf{v}(t, x) d x \\
& +\int_{\mathbb{T}^{N}} F_{u}(x, u(t, x)) u(t, x) h(u(t, x)) d x
\end{aligned}
$$

with $\mathbf{v}(t, x) \equiv(\nabla K \circ u(t,)).(x)$.

Proof. Let $u$ be a classical solution of (1.1). Let $F \in C^{2}\left(\mathbb{T}^{N} \times \mathbb{R}\right)$ be given. Then multiplying (1.1) by $F_{u}(x, u)$ and integrating over $\mathbb{T}^{N}$ leads us to

$$
\begin{aligned}
\frac{d}{d t} \int_{\mathbb{T}^{N}} F(x, u) d x & =\int_{\mathbb{T}^{N}} F_{u}(x, u) \operatorname{div}(u \nabla K \circ u) d x+\int_{\mathbb{T}^{N}} F_{u}(x, u) u h(u) d x \\
& =-\int_{\mathbb{T}^{N}} u \nabla\left[F_{u}(x, u)\right] \mathbf{v} d x+\int_{\mathbb{T}^{N}} F_{u}(x, u) u h(u) d x .
\end{aligned}
$$

Next, one has

$$
u \nabla\left[F_{u}(x, u)\right]=\nabla\left[u F_{u}(x, u)-F(x, u)\right]+\nabla_{x} F(x, u)
$$


which yields

$$
\begin{aligned}
\frac{d}{d t} \int_{\mathbb{T}^{N}} F(x, u) d x= & \int_{\mathbb{T}^{N}}\left[u F_{u}(x, u)-F(x, u)\right] \operatorname{div} \mathbf{v} d x \\
& -\int_{\mathbb{T}^{N}} \nabla_{x} F(x, u) \mathbf{v} d x+\int_{\mathbb{T}^{N}} F_{u}(x, u) u h(u) d x .
\end{aligned}
$$

This completes the proof of the identity for classical solutions and the $C^{2}$ test function. The case of the $C^{1}$ test function is obtained using a limiting argument, and the case of solution integrated along the characteristics can be handled by using a classical regularization procedure.

4. Asymptotic behavior. In this section we investigate the long time behavior of (1.1)-(1.2). In order to overcome the possible lack of asymptotic smoothness of the trajectories, we will use the Young measure framework. First notice that due to Lemma 3.5 (and Lemmas 3.3 and 3.4), for each $n \in \mathbb{Z}^{N}$ the map $t \rightarrow c_{n}[u(t,)$.$] is of$ class $C^{1}$ bounded up to its first derivative (by choosing the test function $F(x, u)=$ $\left.e^{i n x} u\right)$. Next due to the a priori estimate (3.3) one obtains that for each solution

$$
\lim _{t \rightarrow+\infty} c_{n}[u(t, .)]=0 \text { for each } n \in \mathbb{Z}^{N} \backslash\{0\} .
$$

By using the convergence of the Fourier coefficients $c_{n}[u(t,)$.$] it is clear that for a$ given sequence $\left\{t_{k}\right\}_{k \geq 0} \rightarrow+\infty$ one can find a subsequence $t_{k_{p}} \rightarrow+\infty$ and a constant $c \in \mathbb{R}$ such that

$$
u\left(t_{k_{p}}, .\right) \rightarrow c \text { in } L^{2}\left(\mathbb{T}^{N}\right) .
$$

The limit solution may depend on the sequence $\left\{t_{k}\right\}_{k \geq 0}$.

Remark 4.1. From the above remark one concludes that

$$
\lim _{t \rightarrow+\infty}\|\mathbf{v}(t, .)\|_{1, \infty}=0 .
$$

Indeed if $\left\{t_{k}\right\}_{k \geq 0}$ is a given sequence tending to $\infty$, then from the regularity of the kernel (of the class $C^{3}$ ) the sequence $\left\{\mathbf{v}\left(t_{k}, .\right)\right\}_{k \geq 0}$ is bounded in $C_{\sharp}^{2}\left(\mathbb{R}^{N}\right)^{N}$ and thus relatively compact in $C_{\sharp}^{1}\left(\mathbb{R}^{N}\right)^{N}$. Let $\tilde{\mathbf{v}}$ be a limit point of the above sequence in $C_{\sharp}^{1}\left(\mathbb{R}^{N}\right)^{N}$. This means that there exists a subsequence $\left\{t_{k_{p}}\right\}_{p \geq 0}$ such that

$$
\lim _{p \rightarrow \infty} \mathbf{v}\left(t_{n_{p}}, .\right)=\tilde{\mathbf{v}} \text { in } C_{\sharp}^{1}\left(\mathbb{R}^{N}\right)^{N} .
$$

From the above remark, possibly along a subsequence, one may assume that there exists some constant $c \in \mathbb{R}$ such that $u\left(t_{k_{p}},.\right) \rightarrow c$ weakly in $L^{2}\left(\mathbb{T}^{N}\right)$. Hence one gets that for each $x \in \mathbb{T}^{N}$

$$
\int_{\mathbb{T}^{N}} \nabla K(x-y) u\left(t_{k_{p}}, y\right) d y \rightarrow c \int_{\mathbb{T}^{N}} \nabla K(x-y) d y=0 .
$$

This justifies (4.1).

Therefore the goal of this section is to obtain more detailed convergence results on the original distribution $u(t,$.$) . One of the main goals of this section will be to prove$ the weak convergence of $u(t,$.$) to a unique constant distribution as t$ goes to $+\infty$. 
Actually, we will prove a stronger convergence result by showing that there exists a unique constant $E_{\infty}:=\lim _{t \rightarrow+\infty} E[u(t,)$.$] such that$

$$
f(u(t, .)) \rightarrow\left[E_{\infty} f(0)+\left(1-E_{\infty}\right) f(1)\right] \text { in } L^{2}\left(\mathbb{T}^{N}\right) \text { as } t \rightarrow+\infty
$$

for each continuous function $f: \mathbb{R} \rightarrow \mathbb{R}$. We will use this type of convergence result to derive a strong convergence result for some specific initial data.

In order to state our result, let us recall some definitions related to Young measures theory.

Definition 4.2 (Young measure). Let $(X, d)$ be a separable metric space and let $\mathcal{P}(X)$ be the set of probability measures on $(X, d)$. Let $\Omega$ be a given set endowed with a $\sigma$-algebra $\mathcal{A}$. A map $\nu: \Omega \rightarrow \mathcal{P}(X)$ is said to be a Young measure if for each Borel set $B \in \mathcal{B}(X)$ the function $x \rightarrow \nu_{x}(B)$ is measurable from $(\Omega, \mathcal{A})$ into $[0,1]$. The set of all Young measures from $(\Omega, \mathcal{A})$ into $X$ is denoted by $Y(\Omega, \mathcal{A} ; X)$.

Definition 4.3 (narrow convergence topology). Let $(X, d)$ be a separable metric space and let $(\Omega, \mathcal{A}, \mu)$ be a finite measure space. The set $Y(\Omega, \mathcal{A} ; X)$ is endowed with the narrow convergence topology; this topological space is denoted by $(Y(\Omega, \mathcal{A} ; X) ; \mathcal{T})$, which is defined as the weakest topology on $Y(\Omega, \mathcal{A} ; X)$ such that all the functionals

$$
Y(\Omega, \mathcal{A} ; X) \ni \nu \rightarrow \int_{A}\left(\int_{X} \eta(\omega) \nu_{x}(d \omega)\right) \mu(d x) \in \mathbb{R}
$$

for $A \in \mathcal{A}$ and $\eta \in C_{b}(X ; \mathbb{R})$ are continuous. Here $C_{b}(X ; \mathbb{R})$ denotes the space of continuous and bounded maps from $X$ into $\mathbb{R}$.

Remark 4.4. Using the above notation, note that a sequence $\left\{\nu^{n}\right\}_{n \in \mathbb{N}} \subset Y(\Omega, \mathcal{A} ; X)$ narrowly converges to $\nu \in Y(\Omega, \mathcal{A} ; X)$ if and only if for each continuous function $\eta \in C_{b}(X ; \mathbb{R})$ one has

$$
\lim _{n \rightarrow \infty} \int_{X} \eta(\omega) \nu_{x}^{n}(d \omega)=\int_{X} \eta(\omega) \nu_{x}(d \omega)
$$

wherein the above convergence holds for the weak-* topology of $L^{\infty}(\Omega, \mathcal{A} ; \mathbb{R})$ with respect to the parameter $x \in \Omega$. This can also be rewritten for each $\eta \in C_{b}(X ; \mathbb{R})$ and each $\varphi \in L^{1}(\Omega, \mathcal{A} ; \mathbb{R})$ as

$$
\lim _{n \rightarrow \infty} \int_{\Omega} \varphi(x)\left[\int_{X} \eta(\omega) \nu_{x}^{n}(d \omega)\right] \mu(d x)=\int_{\Omega} \varphi(x)\left[\int_{X} \eta(\omega) \nu_{x}(d \omega)\right] \mu(d x) .
$$

In what follows we will denote

$$
Y_{\sharp}\left(\mathbb{R}^{N}, X\right):=Y\left(\mathbb{T}^{N}, \mathcal{B}\left(\mathbb{T}^{N}\right) ; X\right),
$$

and for each $\Omega \in \mathcal{B}(\mathbb{R})$

$$
Y_{\sharp}\left(\Omega \times \mathbb{R}^{N}, X\right)=Y\left(\Omega \times \mathbb{T}^{N}, \mathcal{B}(\Omega) \otimes \mathcal{B}\left(\mathbb{T}^{N}\right) ; X\right) .
$$

The above spaces will always be endowed with the narrow convergence topology. For this reason, in what follows we will not explicitly write down the topology $\mathcal{T}$.

Definition 4.5 (local narrow convergence topology). Let $(X, d)$ be a separable metric space and let $(\Omega, \mathcal{A}, \mu)$ be a finite measure space. The set $Y(\mathbb{R} \times \Omega, \mathcal{B}(\mathbb{R}) \otimes \mathcal{A} ; X)$ is endowed with the local narrow convergence topology denoted by $\mathcal{T}_{\text {loc }}$, which is defined as the weakest topology on $Y(\mathbb{R} \times \Omega, \mathcal{B}(\mathbb{R}) \otimes \mathcal{A} ; X)$ such that all the functionals

$$
Y(\Omega, \mathcal{A} ; X) \ni \nu \rightarrow \int_{I \times A}\left(\int_{X} \eta(\omega) \nu .(d \omega)\right)(d t \otimes \mu) \in \mathbb{R}
$$


for each $I \subset \mathbb{R}$ bounded interval, $A \in \mathcal{A}$, and $\eta \in C_{b}(X ; \mathbb{R})$ are continuous.

In the following we will use the above notion with $(\Omega, \mathcal{A})=\left(\mathbb{T}^{N}, \mathcal{B}\left(\mathbb{T}^{N}\right)\right)$ and $X=[0, \gamma]$ some real interval. Moreover we shall denote by $Y_{\sharp, l o c}\left(\mathbb{R} \times \mathbb{R}^{N} ;[0, \gamma]\right)$ the topological space $Y_{\sharp}\left(\mathbb{R} \times \mathbb{R}^{N} ;[0, \gamma]\right)$ endowed with the local narrow convergence topology $\mathcal{T}_{\text {loc }}$.

Remark 4.6. Let us also recall that since $\left(\mathbb{T}^{N}, \mathcal{B}\left(\mathbb{T}^{N}\right)\right)$ is a countably generated $\sigma$-algebra then the topology $Y_{\sharp}\left(\mathbb{R}^{N}, X\right)$ is metrizable (see, for instance, the monograph of Castaing, Raynaud de Fitte, and Valadier [13]).

Using these definitions, the main result of this section reads as follows.

TheOrem 4.7. Let Assumptions 1.1 and 1.2 be satisfied. Let $u_{0} \in L_{\sharp,+}^{\infty}\left(\mathbb{R}^{N}\right)$ be given and let $u \equiv u(t, x)$ be the solution of (1.1) and (1.2) provided by Theorem 2.4. Recalling definition (3.1) let $E_{\infty} \geq 0$ be the quantity defined by

$$
E_{\infty}:=\lim _{t \rightarrow \infty} E[u(t, .)]
$$

Let us denote by $\gamma=\max \left(\left\|u_{0}\right\|_{\infty}, M_{0}\left(\left\|u_{0}\right\|_{L_{\sharp}^{1}\left(\mathbb{R}^{N}\right)}\right)\right)$ (see Lemma 3.4 for the notation). Then for each $t \geq 0$ the map $x \mapsto \delta_{u(t, x)}$ belongs to $Y_{\sharp}\left(\mathbb{R}^{N},[0, \gamma]\right) ; E_{\infty} \in[0,1]$ and

$$
\lim _{t \rightarrow \infty} \delta_{u(t, .)}=E_{\infty} \delta_{0}+\left(1-E_{\infty}\right) \delta_{1}
$$

wherein the above convergence holds for the narrow convergence topology of $Y_{\sharp}\left(\mathbb{R}^{N}\right.$, $[0, \gamma])$.

Before dealing with the proof of the above theorem, let us state and prove several corollaries of this result. The first one is related to the velocity field $\mathbf{v}$. In particular this provides another of the convergence results stated in Remark 4.1.

COROLlary 4.8. Under the same assumptions and notation as in Theorem 4.7, the velocity field $\mathbf{v}(t, x) \equiv \nabla K \circ u(t,).(x)$ satisfies

$$
\lim _{t \rightarrow \infty} \mathbf{v}(t, x)=0 \text { in } C_{\sharp}^{1}\left(\mathbb{R}^{N}\right) \text {. }
$$

We furthermore have the following point dissipativity estimate:

$$
\limsup _{t \rightarrow \infty}\|u(t, .)\|_{\infty} \leq 1 .
$$

Proof. Note that the convergence (4.2) directly follows from Theorem 4.7. Indeed recall that one has

$$
\mathbf{v}(t, x)=\left|\mathbb{T}^{N}\right|^{-1} \int_{\mathbb{T}^{N}} \nabla K(x-y)\left[\int_{[0, \gamma]} \omega \delta_{u(t, y)}(d \omega)\right] d y .
$$

Hence one obtains using Theorem 4.7 that for each $x \in \mathbb{T}^{N}$

$$
\lim _{t \rightarrow \infty} \mathbf{v}(t, x)=\frac{1-E_{\infty}}{\left|\mathbb{T}^{N}\right|} \int_{\mathbb{T}^{N}} \nabla K(x-y) d y=0 .
$$

In addition, since $K \in C_{\sharp}^{3}\left(\mathbb{R}^{N}\right),\{\mathbf{v}(t, .)\}_{t>0}$ is uniformly bounded in $C^{2}$, which leads us to $\mathbf{v}(t,.) \rightarrow 0$ as $t \rightarrow \infty$ for the topology of $C_{\sharp}^{1}\left(\mathbb{R}^{N}\right)$ and completes the proof of (4.2). Hence recalling the notation introduced in the proof of Lemma 3.4, one has

$$
\frac{d X(t)}{d t}=X(t)\left[\operatorname{div} \mathbf{v}\left(t, \Pi_{v}(t, 0) x\right)+h(X(t))\right] .
$$


Thus since $\|\operatorname{div} \mathbf{v}(t, .)\|_{0, \infty} \rightarrow 0$ as $t \rightarrow \infty$, one obtains that for each $T>0$ and $x \in \mathbb{T}^{N}$

$$
\limsup _{t \rightarrow \infty} u(t, x) \leq \Psi(T):=\inf \left\{z>1: \sup _{t \geq T}\|\operatorname{div} \mathbf{v}(t, .)\|_{0, \infty}+h(y) \leq 0 \forall y \geq z\right\} .
$$

Finally note that because of Assumption 1.1, one has $\Psi(T) \rightarrow 1$ as $T \rightarrow \infty$ and the result follows.

Our second corollary allows us to characterize the asymptotic compactness of the trajectories.

COROLlary 4.9. Under the same assumptions and notation as in Theorem 4.7, let $\left\{t_{n}\right\}_{n \geq 0}$ be a sequence tending to $\infty$ as $n \rightarrow \infty$. Then the sequence $\left\{u\left(t_{n},.\right)\right\} \subset$ $L_{\sharp}^{\infty}\left(\mathbb{R}^{N}\right)$ is relatively compact in $L_{\sharp}^{1}\left(\mathbb{R}^{N}\right)$ if and only if $E_{\infty} \in\{0,1\}$. We furthermore have

$$
E_{\infty}=0 \text { (resp., 1) } \Leftrightarrow \lim _{t \rightarrow \infty} u(t, x)=1 \text { (resp., 0) in } L_{\sharp}^{1}\left(\mathbb{R}^{N}\right) .
$$

This corollary is a direct consequence of Young measure properties (see Corollary 3.1 .5 in [13]).

Our next corollary describes some situations ensuring that $E_{\infty}=0$.

COROLlary 4.10. Under the same assumptions and notation as in Theorem 4.7, let us assume that there exists $\delta>0$ such that $u_{0}(x) \geq \delta$ for a.e. $x \in \mathbb{R}^{N}$. Then one has $E_{\infty}=0$ and $\lim _{t \rightarrow \infty} u(t, x)=1$ in $L_{\sharp}^{1}\left(\mathbb{R}^{N}\right)$.

Proof. Recalling the notation introduced in the proof of Lemma 3.4, one has

$$
\frac{d X(t)}{d t}=X(t)\left[\operatorname{div} \mathbf{v}\left(t, \Pi_{v}(t, 0) x\right)+h(X(t))\right] .
$$

From this one may observe that $u(t, x)>0$ for all $t>0$ and $x \in \mathbb{T}^{N}$. Since $\|\operatorname{div} \mathbf{v}(t, .)\|_{0, \infty} \rightarrow 0$ as $t \rightarrow \infty$, there exists $T>0$ large enough such that

$$
\|\operatorname{div} \mathbf{v}(t, .)\|_{0, \infty} \leq \varepsilon \forall t \geq T,
$$

where $\varepsilon>0$ is a given parameter such that $h(0)-\varepsilon>0$. Now using the above integration along characteristic, one obtains that for each $t \geq T$ and each $x \in \mathbb{T}^{N}$

$$
u(t, x) \geq \phi(t),
$$

wherein $\phi(t)$ is the solution of the ordinary differential equation

$$
\phi^{\prime}(t)=\phi(t)[h(\phi(t))-\varepsilon], t \geq T, \quad \text { and } \quad \phi(T)=\min _{x \in \mathbb{T}^{N}} u(T, x) .
$$

Since $h(0)-\varepsilon>0$, there exist $\eta \in(0,1)$ and $T>0$ such that

$$
u(t, x) \geq \eta \forall t \geq T, x \in \mathbb{T}^{N} .
$$

Let $\tilde{f}:[0, \gamma] \rightarrow \mathbb{R}$ be a given continuous functions such that

$$
\widetilde{f}(s)=0 \text { if } s \geq \eta \text { and } \widetilde{f}(0)=1 .
$$

Then according to Theorem 4.7 one has

$$
\lim _{t \rightarrow \infty} \int_{\mathbb{T}^{N}} \widetilde{f}(u(t, x)) d x=E_{\infty} .
$$


On the other hand, because of (4.3) and the definition of $\widetilde{f}$, one has $\widetilde{f}(u(t,))=$.0 for each $t \geq T$, which implies that $E_{\infty}=1$. Hence $\delta_{u(t, .)} \rightarrow \delta_{1}$ as $t \rightarrow \infty$ and the $L^{1}$ convergence follows from standard Young measure properties (see [13, 27, 28]).

It remains to prove Theorem 4.7. This proof requires several lemmas. Before going into the proof of Theorem 4.7, let us first recall further definitions and notation. Recalling the definition of $\gamma$ in Theorem 4.7 let us consider the probability space $\mathcal{P}\left(\mathbb{T}^{N} \times[0, \gamma]\right)$, and let us recall that the usual weak-* topology on $\mathcal{P}\left(\mathbb{T}^{N} \times[0, \gamma]\right)$ is metrizable using the so-called bounded dual Lipschitz metric defined by

$$
\Theta(\mu, \nu)=\sup \left\{\left|\int_{\mathbb{T}^{N} \times[0, \gamma]} f d(\mu-\nu)\right|, f \in \operatorname{Lip}\left(\mathbb{T}^{N} \times[0, \gamma]\right),\|f\|_{\text {Lip }} \leq 1\right\} .
$$

Recall that the Lipschitz norm is defined in (2.1). Therefore the above distance does not correspond to the 1-Wasserstein distance. We refer to Theorem 18 of Dudley [16] and to the textbook of Billingsley [8] for the equivalence between the weak-* topology on $\mathcal{P}\left(\mathbb{T}^{N} \times[0, \gamma]\right)$ and the topology induced by $\Theta(.,$.$) . In what follows, the$ probability space $\mathcal{P}\left(\mathbb{T}^{N} \times[0, \gamma]\right)$ is always endowed with the metric topology induced by $\Theta$ without further precision.

Let $\left\{t_{n}\right\}_{n \geq 0}$ be a given increasing sequence tending to $\infty$ as $n \rightarrow \infty$. Using the above definition, one will show the following result.

Lemma 4.11. Under the assumptions of Theorem 4.7, let $T>0$ be given. Then the sequence of maps $\mu^{n} \in C^{0}\left([-T, T] ; \mathcal{P}\left(\mathbb{T}^{N} \times[0, \gamma]\right)\right)$ defined by

$$
\mu_{t}^{n}=\left|\mathbb{T}^{N}\right|^{-1} d x \otimes \delta_{u\left(t+t_{n}, x\right)}
$$

is relatively compact in $C^{0}\left([-T, T] ; \mathcal{P}\left(\mathbb{T}^{N} \times[0, \gamma]\right)\right)$. Note that the definition of $\mu_{t}^{n}$ means that for each continuous function $f \in C\left(\mathbb{T}^{N} \times[0, \gamma] ; \mathbb{R}\right)$

$$
\int_{\mathbb{T}^{N} \times[0, \gamma]} f(x, y) d \mu_{t}^{n}(x, y)=\left|\mathbb{T}^{N}\right|^{-1} \int_{\mathbb{T}^{N}} f\left(x, u\left(t+t_{n}, x\right)\right) d x .
$$

Proof. This result is a consequence of the identity provided in Lemma 3.5. Let us first show that there exists some constant $M>0$ such that for each $(t, s) \in[0, \infty)^{2}$

$$
\Theta\left(\left|\mathbb{T}^{N}\right|^{-1} d x \otimes \delta_{u(t, x)},\left|\mathbb{T}^{N}\right|^{-1} d x \otimes \delta_{u(s, x)}\right) \leq M|t-s| .
$$

Let $F \equiv F(x, u) \in C^{1}\left(\mathbb{T}^{N} \times \mathbb{R}\right)$ be given. Let $(t, s) \in[0, \infty)^{2}$ be given such that $s \leq t$. Then Lemma 3.5 yields

$$
\int_{\mathbb{T}^{N}} F(x, u(t, x)) d x-\int_{\mathbb{T}^{N}} F(x, u(s, x)) d x=\int_{s}^{t} \Gamma_{F}(l) d l,
$$

wherein we have set

$$
\begin{aligned}
\Gamma_{F}(t):= & \int_{\mathbb{T}^{N}}\left[u(t, x) F_{u}(x, u(t, x))-F(x, u(t, x))\right] \operatorname{div} \mathbf{v}(t, x) d x \\
& -\int_{\mathbb{T}^{N}} \nabla_{x} F(x, u(t, x)) \mathbf{v}(t, x) d x \\
& +\int_{\mathbb{T}^{N}} F_{u}(x, u(t, x)) u(t, x) h(u(t, x)) d x .
\end{aligned}
$$


On the other hand, since $0 \leq u(t, x) \leq \gamma$ and using the estimates for $\mathbf{v}$ in Lemma 3.3, one directly obtains that there exists some constant $M=M(\gamma)$ such that for all $F \in C^{1}\left(\mathbb{T}^{N} \times \mathbb{R}\right)$ and each $l \in[0, \infty)$

$$
\left|\Gamma_{F}(l)\right| \leq M\|F\|_{\operatorname{Lip}\left(\mathbb{T}^{N} \times[0, \gamma] ; \mathbb{R}\right)} .
$$

Finally (4.5) follows from (4.6) and (4.7).

To complete the proof of the lemma, it is sufficient to notice that Prohorov's compactness theorem (see [8]) implies that $\mathcal{P}\left(\mathbb{T}^{N} \times[0, \gamma]\right)$ is a compact metric space, and the result follows from the Arzelà-Ascoli theorem.

Now using well-known results about Young measures (see, for instance, [13, 27, $28]$ ), since $u$ is uniformly bounded, the following compactness result holds true.

LEMma 4.12. The sequence $\left\{\delta_{u\left(t_{n}, .\right)}\right\}_{n \geq 0}$ is relatively compact for the narrow convergence topology of $Y_{\sharp}\left(\mathbb{R}^{N},[0, \gamma]\right)$.

Using the two above results, namely, Lemmas 4.11 and 4.12, one may assume that, up to a subsequence,

$$
\lim _{n \rightarrow \infty} \mu_{t}^{n}=\mu_{t} \quad \text { and } \quad \lim _{n \rightarrow \infty} \delta_{u\left(t_{n}, x\right)}=\nu_{x}
$$

where the limits hold respectively for the topology of $C_{\text {loc }}^{0}\left(\mathbb{R} ; \mathcal{P}\left(\mathbb{T}^{N} \times[0, \gamma]\right)\right)$ and for the narrow convergence topology of $Y_{\sharp}\left(\mathbb{R}^{N},[0, \gamma]\right)$. Here we would like to recall that the limits $\mu_{t}$ and $\nu_{x}$ depend on the chosen and fixed sequence $\left\{t_{n}\right\}_{n \geq 0}$.

Next, due to (4.4), one has for each continuous function $f \in C\left(\mathbb{T}^{N} \times[0, \gamma] ; \mathbb{R}\right)$ and each $n \geq 0$

$$
\int_{\mathbb{T}^{N} \times[0, \gamma]} f(x, y) d \mu_{0}^{n}(x, y)=\left|\mathbb{T}^{N}\right|^{-1} \int_{\mathbb{T}^{N}} \int_{[0, \gamma]} f(x, y) d \delta_{u\left(t_{n}, x\right)}(y) d x .
$$

Passing to the limit $n \rightarrow \infty$ yields

$$
\int_{\mathbb{T}^{N} \times[0, \gamma]} f(x, y) d \mu_{0}(x, y)=\left|\mathbb{T}^{N}\right|^{-1} \int_{\mathbb{T}^{N}} \int_{[0, \gamma]} f(x, y) d \nu_{x}(y) d x,
$$

for each continuous function $f \in C\left(\mathbb{T}^{N} \times[0, \gamma] ; \mathbb{R}\right)$. This can be rewritten as

$$
\mu_{0}=\left|\mathbb{T}^{N}\right|^{-1} d x \otimes \nu_{x}
$$

Next the following lemma is also a direct consequence of Young measure properties.

Lemma 4.13. The sequence $\left\{\delta_{u\left(t+t_{n}, x\right)}\right\}_{n \geq 0}$ is relatively compact for the local narrow convergence topology of $Y_{\sharp, l o c}\left(\mathbb{R} \times \mathbb{R}^{N} ;[0, \gamma]\right)$.

Using the above result, up to a subsequence, one may assume that there exists a Young measure $\nu \equiv \nu_{t, x} \in Y_{\sharp}\left(\mathbb{R} \times \mathbb{R}^{N} ;[0, \gamma]\right)$ such that

$$
\lim _{n \rightarrow \infty} \delta_{u\left(t_{n}+t, x\right)}=\nu_{t, x} \text { for the topology of } Y_{\sharp, l o c}\left(\mathbb{R} \times \mathbb{R}^{N} ;[0, \gamma]\right) .
$$

As a consequence one has

$$
\mu_{t}=\left|\mathbb{T}^{N}\right|^{-1} d x \otimes \nu_{t, x} \text { for a.e. }(t, x) \in \mathbb{R} \times \mathbb{T}^{N} .
$$

Here let us also recall that $\nu_{t, x}$ depends on the chosen and fixed sequence $\left\{t_{n}\right\}_{n \geq 0}$. 
The aim of the next lemmas is to identify the family of measures $\nu_{t, x}$. Our next result describes first the property of $\nu_{t, x}$.

LEMma 4.14. There exists a measurable map $a: \mathbb{R} \times \mathbb{T}^{N} \rightarrow \mathbb{R}$ such that $0 \leq$ $a(t, x) \leq 1$ for a.e. $(t, x) \in \mathbb{R} \times \mathbb{T}^{N}$ and

$$
\nu_{t, x}=[1-a(t, x)] \delta_{0}+a(t, x) \delta_{1} \text { for a.e. }(t, x) \in \mathbb{R} \times \mathbb{T}^{N} .
$$

Proof. The proof of this lemma relies on the energy functional $E$ (see (3.1)). Let $T>0$ be given. Then (3.3) implies that

$$
\lim _{n \rightarrow \infty} \iint_{[-T, T] \times \mathbb{T}^{N}} \widehat{G}\left(u\left(t_{n}+t, x\right)\right) d t d x=0,
$$

wherein $\widehat{G}:[0, \infty) \rightarrow[0, \infty)$ denotes the continuous function defined as $\widehat{G}(s)=$ $s|\ln (s)||h(s)|$. Recalling the definition of $\mu_{t}^{n}$ in Lemma 4.11, one obtains that for all $n \geq 0$

$$
\frac{1}{\left|\mathbb{T}^{N}\right|} \iint_{[-T, T] \times \mathbb{T}^{N}} \widehat{G}\left(u\left(t_{n}+t, x\right)\right) d t d x=\int_{-T}^{T}\left[\int_{\mathbb{T}^{N} \times[0, \gamma]} \widehat{G}(y) d \mu_{t}^{n}(x, y)\right] .
$$

Combining (4.8), (4.11), and (4.12) one obtains that

$$
\int_{-T}^{T} \int_{\mathbb{T}^{N}}\left[\int_{[0, \gamma]} \widehat{G}(y) d \nu_{t, x}(y)\right] d x d t=0 \quad \forall T>0 .
$$

Since the map $u \mapsto \widehat{G}(u)$ is positive and only vanishes at $u=0$ and $u=1$, one gets that

$$
\operatorname{supp} \nu_{t, x} \subset\{0\} \cup\{1\} \text { for a.e. }(t, x) \in \mathbb{R} \times \mathbb{T}^{N} \text {. }
$$

The above characterization of the support allows us to rewrite this as (see [15])

$$
\nu_{t, x}=\nu_{t, x}(\{0\}) \delta_{0}+\nu_{t, x}(\{1\}) \delta_{1} \text { for a.e. }(t, x) \in \mathbb{R} \times \mathbb{T}^{N} .
$$

Finally set $a(t, x) \equiv \nu_{t, x}(\{1\})$. Recalling that $(t, x) \mapsto \nu_{t, x}$ is measurable with value in $\mathcal{P}([0, \gamma])$, function $a$ is measurable and the result follows.

Our next result reads as follows.

Lemma 4.15. There exists a measurable map $b: \mathbb{R} \rightarrow \mathbb{R}$ such that function $a \equiv a(t, x)$ provided by Lemma 4.14 satisfies $a(t, x) \equiv b(t)$ for a.e. $(t, x) \in \mathbb{R} \times \mathbb{T}^{N}$.

Proof. Here again the proof of this lemma relies on (3.3). Recalling Assumption 1.2 (ii) let us notice that (3.3) yields that for each $\tau>0$

$$
\lim _{n \rightarrow \infty} \int_{-\tau}^{\tau} \sum_{k \in \mathbb{Z}^{N} \backslash\{0\}}\|k\|^{2} c_{k}[K]\left|c_{k}\left[u\left(t+t_{n}, .\right)\right]\right|^{2} d t=0 .
$$

This more particularly implies that for each given $k \in \mathbb{Z}^{N} \backslash\{0\}$ and each $\tau>0$ one has

$$
\lim _{n \rightarrow \infty} \int_{-\tau}^{\tau}\left|c_{k}\left[u\left(t+t_{n}, .\right)\right]\right|^{2} d t=0
$$


Now Hölder inequality yields for each $\varphi \in L_{l o c}^{2}(\mathbb{R})$, for each $\tau>0$, and for each $k \in \mathbb{Z}^{N} \backslash\{0\}$ that

$$
\lim _{n \rightarrow \infty} \int_{-\tau}^{\tau} \varphi(t) c_{k}\left[u\left(t+t_{n}, .\right)\right] d t=0 .
$$

Using the definition of $\nu_{t, x}$ in (4.10) and its description in Lemma 4.14 one obtains that for each $\tau>0$ and for each $k \in \mathbb{Z}^{N} \backslash\{0\}$

$$
\lim _{n \rightarrow \infty} \int_{-\tau}^{\tau} \varphi(t) c_{k}\left[u_{n}(t, .)\right] d t=\int_{-\tau}^{\tau} \varphi(t) c_{k}[a(t, .)] d t=0
$$

This implies that $c_{k}[a(t,)]=$.0 for all $k \in \mathbb{Z}^{N} \backslash\{0\}$ for a.e. $t \in \mathbb{R}$, and the result follows.

In order to complete the proof of Theorem 4.7, we will prove the following lemma. LEMma 4.16. The following hold true: $E_{\infty} \in[0,1]$ and $b(t) \equiv 1-E_{\infty}$ for a.e. $t \in \mathbb{R}$.

Proof. The proof of this result is based on the identity derived in Lemma 3.5 with $F(x, s) \equiv s$ applied with $u\left(t+t_{n}, x\right)$, which for each $n \geq 0$ and each $t>-t_{n}$ reads as

$$
\partial_{t} \int_{\mathbb{T}^{N}} u\left(t+t_{n}, x\right) d x=\int_{\mathbb{T}^{N}} u\left(t+t_{n}, x\right) h\left(u\left(t+t_{n}, x\right)\right) d x .
$$

Let $\varphi \in C_{c}^{1}(\mathbb{R})$ be given. Multiplying the above equation by $\varphi$ for each $n \geq 0$ large enough such that $\operatorname{supp} \varphi \subset\left[-t_{n}, \infty\right)$ and integrating over $\mathbb{R}$ leads to

$$
-\int_{\mathbb{R} \times \mathbb{T}^{N}} \varphi^{\prime}(t) u\left(t+t_{n}, x\right) d t d x=\int_{\mathbb{R}} \varphi(t) \int_{\mathbb{T}^{N}}\left[\int_{[0, \gamma]} f(z) d \delta_{u\left(t+t_{n}, x\right)}(z)\right] d x d t .
$$

Letting $n \rightarrow \infty$ and using Lemma 4.15 leads us to the following: for all $\varphi \in C_{c}^{1}(\mathbb{R})$,

$$
\int_{\mathbb{R}} \varphi^{\prime}(t) b(t) d t=\int_{\mathbb{R}} \varphi(t) \int_{\mathbb{T}^{N}}\left[\int_{[0, \gamma]} f(z)\left((1-b(t)) \delta_{0}+b(t) \delta_{1}\right)(d z)\right] d x d t=0 .
$$

This implies that there exists some constant $b^{*} \in[0,1]$ such that $b(t) \equiv b^{*}$.

Finally, recalling that $E\left[u\left(t+t_{n},.\right)\right] \rightarrow E_{\infty}$ as $n \rightarrow \infty$ for each $t \in \mathbb{R}$ directly yields $E_{\infty}=1-b^{*}$, and the result follows.

To complete the proof of Theorem 4.7 note that we infer from the above lemmas that $E_{\infty} \in[0,1]$, while $\nu_{t, x}=E_{\infty} \delta_{0}+\left(1-E_{\infty}\right) \delta_{1}$ for a.e. $(t, x) \in \mathbb{R} \times \mathbb{T}^{N}$. Recalling that function $t \rightarrow \mu_{t}$ defined in (4.8) is continuous from $\mathbb{R}$ into $\mathcal{P}\left(\mathbb{T}^{N} \times[0, \gamma]\right)$ and that (4.11) implies that for all $t \in \mathbb{R}$

$$
\mu_{t} \equiv\left|\mathbb{T}^{N}\right|^{-1} d x \otimes\left(E_{\infty} \delta_{0}+\left(1-E_{\infty}\right) \delta_{1}\right) .
$$

Finally recalling (4.9) one obtains that

$$
\nu_{x} \equiv E_{\infty} \delta_{0}+\left(1-E_{\infty}\right) \delta_{1} \text { for a.e. } x \in \mathbb{T}^{N} .
$$

Since the sequence $\left\{t_{n}\right\}_{n \geq 0}$ denotes any sequence increasing tending to infinity, recalling the definition of $x \mapsto \nu_{x}$ in (4.8), Lemma 4.12, and Remark 4.6 this completes the proof of Theorem 4.7. 
5. Concluding remarks. In this work we have given a detailed description of the asymptotic behavior of system (1.1)-(1.2) (see Theorem 4.7). The situation $E_{\infty} \in$ $(0,1)$ remains a pathological behavior (see Corollary 4.9). However, we conjecture that as soon as $u_{0} \not \equiv 0$ then the corresponding solution $u \equiv u(t, x)$ strongly converges to 1 (namely, $E_{\infty}=0$ ). This means that the Lebesgue measure of the set $Z_{t}=\{x \in$ $\left.\mathbb{T}^{N}: u(t, x)=0\right\}$ tends to zero as $t$ goes to $\infty$. However, we are not able to prove this result for general initial data (see Corollary 4.10).

Let us finally notice that even if such a pathological behavior $\left(E_{\infty} \in(0,1)\right)$ occurs, then it is "unstable" with respect to diffusive perturbation. Indeed if we consider the equation

$$
\partial_{t} u(t, x)=\Delta u(t, x)+\operatorname{div}(u(t, x)(K \circ u(t, .))(x))+f(u(t, x))
$$

on $\mathbb{T}^{N}$, supplemented together with some initial data $u_{0} \in L_{\sharp,+}^{\infty}\left(\mathbb{R}^{N}\right)$, then it is easy to see that under the regularity Assumption 1.2 (i) for the kernel $K$ the solution goes to 1 uniformly as $t \rightarrow \infty$ as soon as $u_{0} \not \equiv 0$. To see this, let us first notice that using the strong maximum principle one has $u(t, x)>0$ for all $t>0$ and $x \in \mathbb{T}^{N}$. Next uniform bound of the solution follows from arguments similar to those in Lemma 3.2 coupled with the maximum principle. Because of the uniform bound of the solution, the convection term is also uniformly bounded, and the usual parabolic regularity applies and ensures that the solution is asymptotically relatively compact in $C\left(\mathbb{T}^{N}\right)$. Moreover the functional $t \rightarrow E[u(t,)$.$] defined in (3.1) is decreasing along$ the trajectories and satisfies a property similar to that in (3.3), which reads as

$$
\begin{aligned}
\frac{d}{d t} E[u(t, .)]= & -\frac{1}{\left|\mathbb{T}^{N}\right|} \int_{\mathbb{T}^{N}}\left[\frac{|\nabla u|^{2}}{u}+u|\ln u||h(u)|\right] d x \\
& +\frac{1}{\left|\mathbb{T}^{N}\right|^{2}} \int_{\mathbb{T}^{N} \times \mathbb{T}^{N}} u(x) \Delta K(x-y) u(y) d x d y .
\end{aligned}
$$

Recalling that Assumption 1.2 (ii) ensures that the last term in the above equality is negative, one directly obtains the convergence to 1 for the solution.

Acknowledgment. The authors would like to thank the anonymous referees for their valuable comments and suggestions.

\section{REFERENCES}

[1] N. J. Armstrong, K. J. Painter, and J. A. Sherratt, A continuum approach to modelling cell-cell adhesion, J. Theoret. Biol., 243 (2006), pp. 98-113.

[2] A. J. Bernoff and C. M. Topaz, A primer of swarm equilibria, SIAM J. Appl. Dyn. Syst., 10 (2011), pp. 212-250; reprint appears in SIAM Rev., 55 (2013), pp. 709-747.

[3] J. Bedrossian, N. Rodriguez, And A. L. Bertozzi, Local and global well-posedness for aggregation equations and Patlak-Keller-Segel models with degenerate diffusion, Nonlinearity, 24 (2011), pp. 1683-1714.

[4] A. L. Bertozzi, J. A. Carrilo, and T. Laurent, Blowup in multidimensional aggregation equations with mildly singular interaction kernels, Nonlinearity, 22 (2009), pp. 683-710.

[5] A. L. Bertozzi, J. B. Garnett, and T. Laurent, Characterization of radially symmetric finite time blowup in multidimensional aggregation equations, SIAM J. Math. Anal., 44 (2012), pp. 651-681.

[6] A. L. Bertozzi And J. Brandman, Finite-time blow-up of $L^{\infty}$-weak solutions of an aggregation equation, Commun. Math. Sci., 8 (2010), pp. 45-65.

[7] A. L. Bertozzi, T. Laurent, and J. Rosado, $L^{p}$ theory for the multidimensional aggregation equation, Comm. Pure Appl. Math., 64 (2011), pp. 45-83.

[8] P. Billingsley, Convergence of Probability Measures, 2nd ed., Wiley, New York, 1999. 
[9] M. Bodnar and J. J. L. Velazquez, Derivation of macroscopic equations for individual cellbased models. A formal approach, Math. Methods Appl. Sci., 28 (2005), pp. 1757-1779

[10] M. Bodnar and J. J. L. VelazQuez, An integro-differential equation arising as a limit of individual cell-based models, J. Differential Equations, 222 (2006), pp. 341-380.

[11] M. Burger And M. Di Francesco, Large time behavior of nonlocal aggregation models with nonlinear diffusion, Netw. Heterog. Media, 3 (2008), pp. 749-785.

[12] V. Capasso and D. Morale, Asymptotic behavior of a system of stochastic particles subject to nonlocal interactions, Stoch. Anal. Appl., 27 (2009), pp. 574-603.

[13] C. Castaing, P. Raynaud de Fitte, and M. Valadier, Young Measures on Topological Spaces: With Applications in Control Theory and Probability Theory, Springer, 2004.

[14] L. Chayes and V. Panferov, The McKean-Vlasov equation in finite volume, J. Stat. Phys., 138 (2010), pp. 351-380.

[15] J. Dieudonné, Treatise on Analysis, Vol. 2, Academic Press, New York, 1976.

[16] R. M. Dudley, Convergence of Baire measures, Studia Math., 27 (1966), pp. 251-268.

[17] A. Ducrot, F. Le Foll, P. Magal, H. Murakawa, J. Pasquier, and G. F. Webb, An in vitro cell population dynamics model incorporating cell size, quiescence, and contact inhibition, Math. Models Methods Appl. Sci., 21 (2011), pp. 871-892.

[18] T. Hillen, K. J. Painter, And M. Winkler, Convergence of a cancer invasion model to a logistic chemotaxis model, Math. Models Methods Appl. Sci., 23 (2013), pp. 165-198.

[19] A. J. Leverentz, C. M. Topaz, and A. J. Bernoff, Asymptotic dynamics of attractiverepulsive swarms, SIAM J. Appl. Dyn. Syst., 8 (2009), pp. 880-908.

[20] A. Majda And A. Bertozzi, Vorticity and Incompressible Flow, Cambridge University Press, Cambridge, UK, 2002.

[21] A. Mogilner and L. Edelstein-Keshet, A non-local model for a swarm, J. Math. Biol., 38 (1999), pp. 534-570.

[22] D. Morale, V. Capasso, and K. Oelschläger, An interacting particle system modelling aggregation behavior: From individuals to populations, J. Math. Biol., 50 (2005), pp. 4966.

[23] K. Oelschläger, A law of large numbers for moderately interacting diffusion processes, Z. Wahrsch. Verw. Gebiete, 69 (1985), pp. 279-322.

[24] K. OELSChläGER, Large systems of interacting particles and the porous medium equation, J. Differential Equations, 88 (1990), pp. 294-346.

[25] B. Perthame and A. L. Dalibard, Existence of solutions of the hyperbolic Keller-Segel model, Trans. Amer. Math. Soc., 361 (2009), pp. 2319-2335.

[26] G. RAoul, Non-local interaction equations: Stationary states and stability analysis, Differential Integral Equations, 25 (2012), pp. 417-440.

[27] D. SERRE, Systèmes de lois de conservation. I, Diderot Editeur, Paris, 1996.

[28] D. Serre, Systèmes de lois de conservation. II, Diderot Editeur, Paris, 1996. 